\title{
光致噻吨酮与胺类、酚类、醇类的电子转移和氢转移反应
}

\author{
孙倩 王金婷张立敏* 杨茂萍 \\ (中国科学技术大学化学物理系, 合肥 230026)
}

\begin{abstract}
摘要: 利用激光闪光光解方法研究了一系列胺类、酚类、醇类在脱氧乙腈中猝灭噻吨酥 $(\mathrm{TX})$ 三重态的反应, 得 到了相应的瞬态吸收光谱和猝灭速率常数 $\left(k_{q}\right)$. 通过对光谱演变特性的分析, 推断出三重态噻吨酮与不含有活泼 氢的胺发生了电子转移反应, 与含有活泼氢的胺发生了电子-质子转移反应. 三重态噻吨酮与酚类、醇类反应中 观察到噻吨酮加氢自由基的生成, 据此推断出三重态噻吨酮与酚类、醇类发生了氢转移反应. 胺类的猝灭速率常 数随着反应自由能变 $(\Delta G)$ 的增大而减小, 说明电子转移影响了噻吨酮三重态的猝灭. 酚类的猝灭速率常数先随 $\Delta G$ 增大而减小, 后随酚阳离子的酸性增强逐渐增大, 可能是猝灭过程中电子转移影响减弱的同时氢转移影响逐 渐增强. 醇类的猝灭速率常数随着醇的 $\alpha-\mathrm{C}-\mathrm{H}$ 键能的增大而减小, 说明 $\alpha-\mathrm{C}-\mathrm{H}$ 键能是影响噻吨酮三重态猝灭 的关键因素. 比较以前研究的胺类、酚类、醇类与三重态呫吨酮 $(\mathrm{XT})$ 、芴酮(FL)反应的结果可知, 由于分子结构差 异性的影响, 相关的猝灭速率常数按照师吨酮、噻吨酮、荡酮的顺序逐渐减小.
\end{abstract}

关键词: 电子转移; 氢转移; 噻吨酮; 胺类 酚类; 醇类

中图分类号: 0644

\section{Photoinduced Electron and Hydrogen Transfer Reactions of Thioxanthone with Amines, Phenols and Alcohols}

\author{
SUN Qian $\quad$ WANG Jin-Ting $\quad$ ZHANG Li-Min* $\quad$ YANG Mao-Ping \\ (Department of Chemical Physics, University of Science and Technology of China, Hefei 230026, P. R. China)
}

\begin{abstract}
The quenching reactions of triplet thioxanthone (TX) by a series of amines, phenols, and alcohols were investigated by laser flash photolysis in deoxygenated acetonitrile. We obtained corresponding transient absorption spectra and quenching rate constants $\left(k_{q}\right)$. From changes in the transient absorption spectra, we determine that the electron transfer reactions occur between triplet TX and amines without an active hydrogen while electron/proton transfer reactions occur between triplet TX and amines with an active hydrogen. The appearance of hydrogenated radicals can be regarded as evidence for hydrogen transfer reactions in the TX/phenol and TX/alcohol systems. In the TX/amine systems, the quenching rate constants decreased with an increase in the free energy change $(\Delta G)$. This indicates that electron transfer reactions influence the quenching of triplet TX. In the TX/phenol systems, the quenching rate constants decreased with an increase in $\Delta G$ firstly, then increased with an increase in phenol cation acidity. This can be explained by considering that charge transfer and hydrogen transfer may play separate but important roles. In the TX/ alcohol system, the quenching rate constants decreased with an increase in the $\alpha-\mathrm{C}-\mathrm{H}$ bonding energy of alcohols, and this indicates that the $\alpha-\mathrm{C}-\mathrm{H}$ bonding energy is a key factor during triplet TX quenching. By comparison with previous studies about the quenching reactions of triplet xanthone (XT) and fluorenone (FL) by a series of amines, phenols, and alcohols, it is established that because of a discrepancy in molecular configurations the quenching rate constants decrease according to the following order: XT, TX, and FL.
\end{abstract}

Key Words: Electron transfer; Hydrogen transfer; Thioxanthone; Amines; Phenols; Alcohols

Received: April 12, 2010; Revised: May 24, 2010; Published on Web: July 19, 2010.

*Corresponding author. Email: 1mzha@ustc.edu.cn; Tel: +86-551-3601119.

The project was supported by the National Natural Science Foundation of China (20673108).

国家自然科学基金(20673108)资助项目

C Editorial office of Acta Physico-Chimica Sinica 
芳香酮是光化学和光生物反应的重要物质, 一 直受到实验和理论计算的高度重视 ${ }^{[1-4]}$. 光致胺类还 原芳香酮的研究被报道得较多 ${ }^{[5-9]}$, 并且胺类、酚类、 醇类与芳香酮的氢转移反应也被广泛地研究过 ${ }^{[10]}$, 此类氢转移反应产生的自由基常作为光聚合反应的 引发剂应用于工业生产 [. 噻吨酮(TX)及其衍生物 的光敏活性在治疗肿瘤方面也有重要的应用, 是一 类在医药方面有重要发展前景的芳香酮 ${ }^{[11-12]}$.

最低三重态的电子结构是影响酮类化合物光致 还原反应的重要因素. 最低三重态电子结构为 $(\pi$, $\pi^{*}$ )的酮类化合物容易发生电子转移反应, 而最低三 重态电子结构为 $\left(n, \pi^{*}\right)$ 的酮类化合物更容易发生 氢转移反应 ${ }^{[13]}$. 噻吨酮最低三重态电子结构为 $(\pi$, $\left.\pi^{*}\right)^{[14]},\left(\pi, \pi^{*}\right)$ 和较低能量的 $\left(n, \pi^{*}\right)$ 容易发生电子-振 动耦合 ${ }^{[15]}$, 使激发三重态的噻吨酮同时具有电子转 移和抽氢的能力. 噻吨酮单重电子态和三重电子态 系间宫跃系数较高 ${ }^{[2]}$, 较高的三重态产率有利于提 高反应产生的自由基的浓度, 便于检测, 所以噻吨 酮是进行这类研究的重要分子. 虽然利用激光闪光 光解的方法研究噻吨酮与胺、醇、酚的反应已有报 道 ${ }^{[-6,65-17]}$, 但据我们所知, 噻吨酮与这三类物质系列 性反应机理, 尤其是猝灭常数的规律性研究, 还没有 被报道过.

本文的工作是利用激光闪光光解方法研究一系 列的胺类、酚类、醇类与三重态噻吨酮的反应. 通过 时间分辨瞬态吸收光谱的变化, 得到了相应的电子 和氢转移产物的吸收峰, 从吸收峰随时间的变化 推测出相关的反应机理. 研究了实验得到的猝灭 速率常数与自由能变、酚阳离子酸性 ${ }^{[18]} 、 \alpha-\mathrm{C}-\mathrm{H}$ 键 能 ${ }^{[19]}$ 的关系. 并且与以前研究的胺类、酚类、醇类与 呫吨酮 $(\mathrm{XT})$ 、芴酮(FL) 三重态反应的结果 ${ }^{[20]}$ 进行了 比较.

\section{1 实验部分}

\section{1 仪器和设备}

本实验采用纳秒级的激光闪光光解装置. $\mathrm{Nd}$ : YAG 激光器 (GCR-170 型, 美国 Spectra Physics 公 司, 重复率 $10 \mathrm{~Hz}$, 光脉宽 $8 \mathrm{~ns}$ )经过三倍频的 $355 \mathrm{~nm}$ 的激光作为光敏剂分子的激发光源. 波长范围为 $300-900 \mathrm{~nm}$ 的 $500 \mathrm{~W}$ 的高压氙灯 (XQ500 型, 中国 电型器材公司)作为分析光源. 分析光与激发光成垂 直方向照射到光程 $10 \mathrm{~mm}$ 的石英池上, 经过聚焦 镜, 通过单色仪(WDG3-3, 长春时代光电有限责任公
司)分光, 再由光电倍增管(GDB59)将光信号转换 为电信号并放大后, 用数字示波器 (400 MHz, TDS380 型, 美国 Tektronix 公司)记录存储瞬态衰减曲线 ${ }^{[21-22]}$. 瞬态吸收光谱通过 A/D 卡 $(50 \mathrm{MHz}, \mathrm{SR} 245$ 型, 美国 Stanford Research Systems 公司)采集得到. 根据中间 体的寿命选择若干合适的采集时间门, A/D 卡在每 个时间门通过信号积分得到了不同时间吸收的强 度, 在有激光照射的情况下扫描单色仪波长可以获 得各个时间门的瞬态吸收光谱(需减去无激光照射 时的背景), 通过对吸收光谱不同的吸收峰进行归属 可以得到瞬态中间体自由基的信息. 试剂除氧后通 过恒流洜(HL-1S 型, 上海沪西分析仪器厂)到达石 英循环池, 以保证样品溶液不被过度照射, 使实验结 果更准确可靠.

\section{2 试剂和样品}

噻吨酮(TX) (98\%)购自美国 Alfa Aesar 公司. $3,5, N, N$-四甲基苯胺 $(\mathrm{TMA})(\geqslant 97 \%)$,三苯胺 (TPA) $(\geqslant 98 \%)$, 4-甲基- $N, N$-二甲基苯胺(DMT)( $\geqslant 99 \%)$, 购 自美国 Fluka 公司. 二苯胺(DPA) $(\geqslant 98 \%) 、 4$-苯基苯 酚(99\%)、4-溴苯酚(99\%)购自美国 Aldrich 公司. 三 乙烯胺 $(\mathrm{DABCO})(\geqslant 99 \%)$ 购自美国 Sigma 公司. $\beta$-胡 萝卜素( $>97.0 \%)$ 购自德国 Merck 公司. 以上药品均 未经进一步纯化直接使用. $N, N$-二甲基苯胺(DMA)、 2-苯乙醇在使用前重新蒸馏两次. 对苯二酚、间苯二 酚、4-甲氧基苯酚、4-叔丁基苯酚、4-氯苯酚, 使用前 均用标准方法提纯. 苯胺(AN)、苯酚、苯甲醇、乙醇、 甲醇为分析纯, 未经进一步纯化而直接使用. 作为溶 剂的乙腈为高效液相色谱纯级 $(\geqslant 99.9 \%)$. 样品在光 照之前通人高纯氩气(99.99\%)20 min 以除去氧气, 所有实验均在室温下完成.

\section{2 结果与讨论}

\section{1 三重态噻吨酮的瞬态吸收光谱及自猝灭速率 常数}

用波长为 $355 \mathrm{~nm}$ 、脉宽为 $8 \mathrm{~ns}$ 、脉冲能量为 $4 \mathrm{~mJ}$ 的脉冲激光照射脱氧乙腈中浓度为 $0.4 \mathrm{mmol} \cdot \mathrm{L}^{-1}$ 的 噻吨酮, 得到其瞬态吸收光谱, 如图 1(a)所示, 可以 看到最大吸收峰为 $620 \mathrm{~nm}$, 此峰在 $0.4 \mu \mathrm{s}$ 时达到最 大, 随着时间延长逐渐减小, $35.0 \mu \mathrm{s}$ 时已经基本衰 减完全. 为了归属此峰, 我们在乙腈中加入噻吨酮和 不同浓度的 $\beta$-胡萝卜素, 经光照射后, 在 $520 \mathrm{~nm}$ 处 有新物质的吸收, 并随着 $\beta$-胡萝卜素的浓度增加吸 收增大, 如图 1(b)所示. 根据前人报道 ${ }^{[23]}, 520 \mathrm{~nm}$ 为 
$\beta$-胡夢卜素三重态的特征吸收, $\beta$-胡萝卜素三重态 能量较低 $\left(85 \mathrm{~kJ} \cdot \mathrm{mol}^{-1}\right)$, 容易发生能量转移反应, 我 们判断其发生了三重态-三重态( T-T)能量转移反 应. 因此, 我们把 $620 \mathrm{~nm}$ 的峰归属为噻吨酮三重态 $\left({ }^{3} \mathrm{TX}\right)$ 的特征吸收峰. 关于噻吨酮三重态吸收峰的 位置, 前人也做了大量工作, 我们得到的结果与 Yates $^{[6]}$ 和 Corrales ${ }^{[24]}$ 等的结果是一致的. 三重态噻吨 酮随着时间的衰减遵循一级反应动力学规律, 如图 2(a) 所示.

单一浓度下激发三重态噻吨酮的猝灭速率由其 随时间变化的衰减曲线单指数拟合得到,一般来说, 噻吨酮浓度越大猝灭速率也越大. 图 2(b)给出了不 同噻吨酮浓度下的自猝灭速率, 猝灭速率常数计算 公式为:

$$
k_{0}=1 / \tau_{0}+k_{\mathrm{sq}} c_{\mathrm{TX}}
$$

其中, $k_{0}$ 为没有猝灭剂时不同浓度噻吨酮三重态的
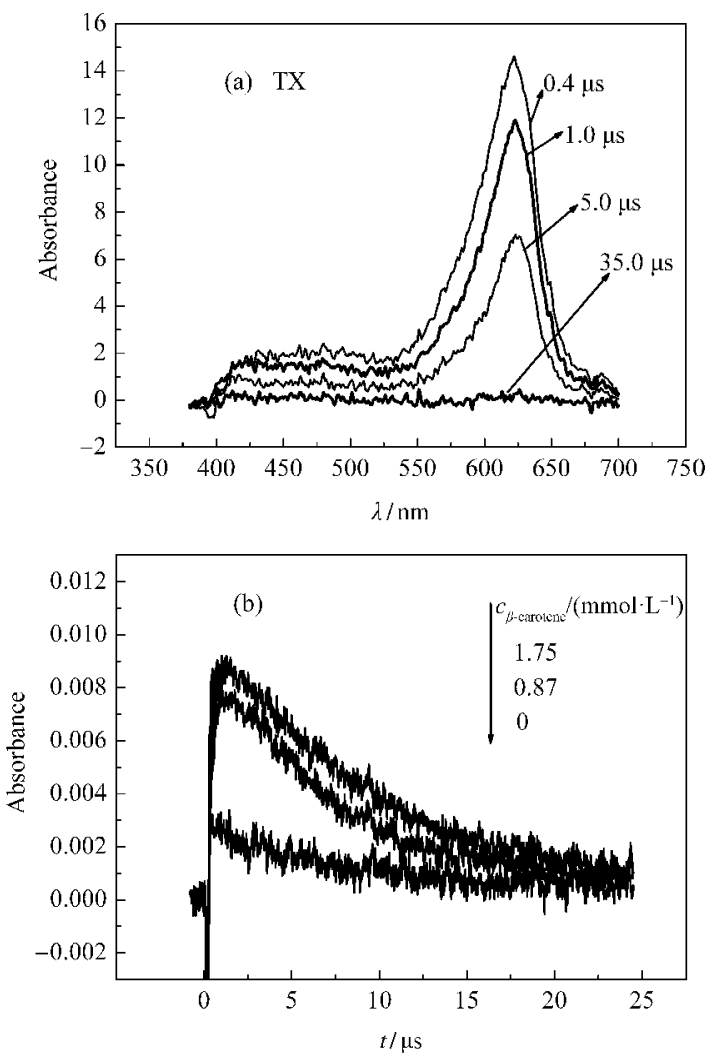

图 $1355 \mathrm{~nm}$ 激光照射脱氧乙腈中(a) TX (0.4 mmol • $\left.\mathrm{L}^{-1}\right)$ 的瞬态吸收光谱及 $(b) \mathrm{TX}\left(0.2 \mathrm{mmol} \cdot \mathrm{L}^{-1}\right)$ 与 $\beta$-胡萝卜 素 $520 \mathrm{~nm}$ 的衰减曲线

Fig.1 (a) Transient absorption spectra of TX $(0.4$ $\left.\mathrm{mmol} \cdot \mathrm{L}^{-1}\right)$ and $(\mathrm{b})$ decay time profiles of $\mathrm{TX}(0.2$ $\left.\mathrm{mmol} \cdot \mathrm{L}^{-1}\right)$ and $\beta$-carotene at $520 \mathrm{~nm}$ irradiated with $355 \mathrm{~nm}$ laser pulse in deoxygen acetonitrile TX: thioxanthone
自猝灭速率, $\tau_{0}$ 为噻吨酮激发三重态的固有寿命, $k_{\mathrm{sq}}$ 为噻吨酮的自猝灭速率常数. 在此实验条件下, 我们得出了 $\tau_{0}$ 和 $k_{\mathrm{sq}}$ 的值分别为 $10.3 \mu \mathrm{s}$ 和 $3.0 \times 10^{7}$ $\mathrm{L} \cdot \mathrm{mol}^{-1} \cdot \mathrm{s}^{-1}$.

\section{2 双分子反应速率常数}

激发三重态的噻吨酮反应活性大大增强, 可以 与容易失去电子和氢原子的物质发生电子转移和 氢转移反应. 本实验选择 $620 \mathrm{~nm}$ 作为分析波长, 通 过猝灭速率对猝灭剂的浓度作图, 并由下式对数据 进行拟合, 得到的直线斜率即为双分子猝灭速率常 数 $k_{\mathrm{q}}$.

$$
k_{\mathrm{obs}}=k_{0}+k_{\mathrm{q}} c_{\mathrm{Q}}
$$

其中, $k_{\mathrm{obs}}$ 代表有猝灭剂时的噻吨酮三重态的猝灭速 率, $c_{\mathrm{Q}}$ 代表猝灭剂浓度. 由(2)式我们可以得到胺类、 酚类、醇类分别猝灭噻吨酮三重态的速率常数.

\section{3 三重态噻吨酮与胺类发生电子-质子转移反应}
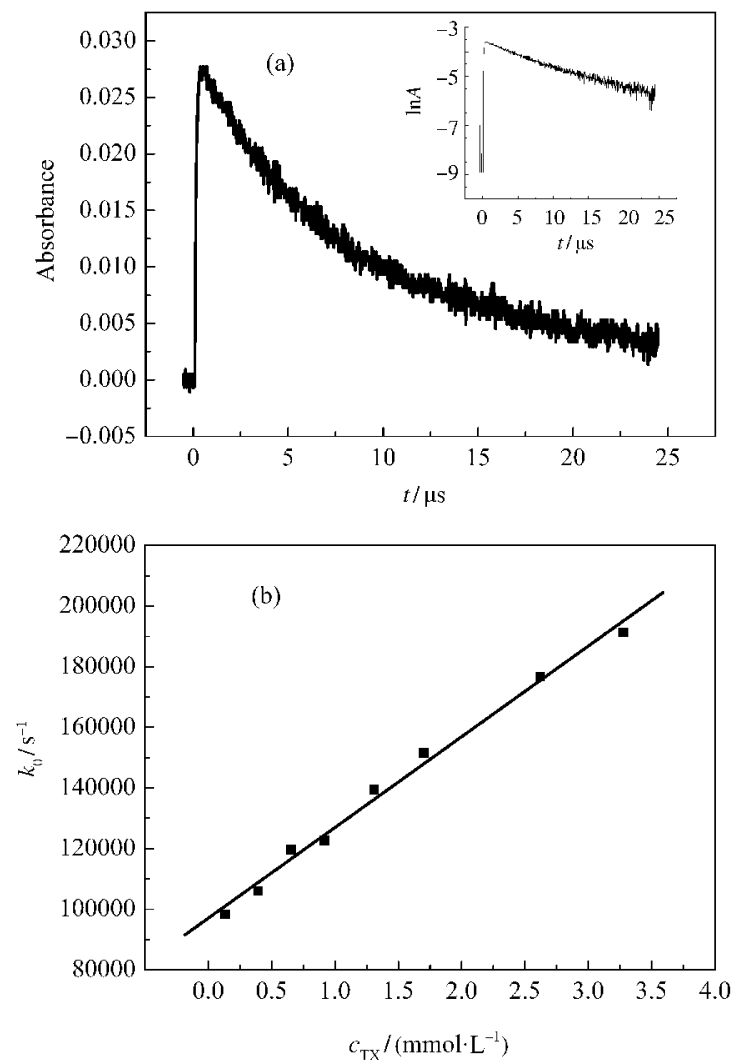

图 $2355 \mathrm{~nm}$ 激光照射脱氧乙腈中(a) TX $\left(0.4 \mathrm{mmol} \cdot \mathrm{L}^{-1}\right)$ $620 \mathrm{~nm}$ 的衰减曲线及 $(\mathrm{b}) \mathrm{TX}$ 的自猝灭速率与浓度的关系 Fig.2 (a) Decay time profiles of $\mathrm{TX}\left(0.4 \mathrm{mmol} \cdot \mathrm{L}^{-1}\right)$ at $620 \mathrm{~nm}$ band and (b) the relationship between the self quenching rate and $T X$ concentration irradiated with $355 \mathrm{~nm}$ laser pulse in deoxygen acetonitrile Inset in Fig.2(a) shows the logarithmic plots of absorbance $(\ln A) v s$ time. 
三重态噻吨酩与胺类的电子转移反应前人的研 究已有所涉及 ${ }^{[5-6,16]}$. 本实验通过噻吨酮和胺类反应 的瞬态吸收光谱得到相应自由基的吸收峰, 为胺类 和噻吨酮反应通过电子转移或电子-质子转移的机 理提供了强有力的证据. TPA 是一种经常用来提供 电子的胺, 噻吨酮/乙腈中加人一定浓度的 TPA, 得 到如图 3(a)所示的瞬态吸收光谱, 可以看到伴随着 噻吨酮三重态吸收峰的衰减, 在 $550 \mathrm{~nm}$ 处有一个 新的吸收峰生成. 根据前人的报道, 这个峰归属为三 苯胺阳离子自由基 $\left(\mathrm{TPA}^{+\bullet}\right)^{[25]}$. 同时, $685 \mathrm{~nm}$ 处也有 一个较小的吸收峰生成, 我们将它归属为噻吨酮阴 离子自由基 $\left(\mathrm{TX}^{-}{ }^{\bullet}\right)$. Scigalski 等 ${ }^{[16}$ 曾报道过噻吨酮阴 离子自由基的吸收为 $680 \mathrm{~nm}$. 根据前人报道 ${ }^{[25]}$,三 苯胺阴离子在 $650 \mathrm{~nm}$ 处也有吸收, 由于噻吨酮三 重态在 $650 \mathrm{~nm}$ 处有一个吸收较大的背景, 我们并 没有观察到明显的瞬态吸收峰. TPA 有一个大 $\pi$ 键, 不容易被抽氢, 实验中没有观察到噻吨酮加氢自 由基的生成. 与 TPA 类似, $\mathrm{DABCO}$ 的 $\mathrm{C}-\mathrm{H}$ 键能较 大, 不容易断键, 只发生了电子转移反应 ${ }^{[5]}$. 据此, 我 们推测噻吨酮和 TPA、DABCO 发生了如下的光引 发电子转移反应:

\section{${ }^{3} \mathrm{TX}^{*}+\mathrm{D} \longrightarrow{ }^{3}\left(\mathrm{TX}^{-\bullet} \cdots \mathrm{D}^{+\bullet}\right) \rightarrow \mathrm{TX}^{-\bullet}+\mathrm{D}^{\bullet}$}

实验得到了 DMT、TMA 与噻吨酮在乙腈中反 应的瞬态吸收光谱. 在图 3(b)所示的 TX/DMT 的瞬 态吸收光谱中可以看到, 随着噻吨酮三重态的衰减, 在 $420 、 475$ 和 $685 \mathrm{~nm}$ 分别有三个新的吸收峰生成. $420 \mathrm{~nm}$ 的峰应归属为噻吨酮加氢自由基 $\left(\mathrm{TXH}^{*}\right)^{[6]}$, $475 \mathrm{~nm}$ 的峰归属为 DMT 阳离子自由基 $\left(\mathrm{DMT}^{\circ}{ }^{\bullet}\right.$ ), $685 \mathrm{~nm}$ 的峰为噻吨酮阴离子自由基. 在图 3(c)所示 的 TX/TMA 的瞬态吸收光谱中, 我们得到了与 TX/ DMT 类似的结果, 可以看到随着噻吨酮三重态吸收
峰的衰减, 在 $420 、 495$ 和 $685 \mathrm{~nm}$ 处得到了三个新 的吸收峰, 这三个峰分别归属为噻吨酮加氢自由基, TMA 阳离子自由基 $\left(\mathrm{TMA}^{+\bullet}\right)$, 噻吨酮阴离子自由基. Pan 等 ${ }^{[13]}$ 研究认为 $\mathrm{DMT}^{+} \cdot$ 和 $\mathrm{TMA}^{+} \cdot$ 的特征吸收峰 分别为 480 和 $510 \mathrm{~nm}$. 由此, 我们判断以上反应产 生了相应的阴阳离子和加氢自由基.

我们也测量了 $\mathrm{AN} 、 \mathrm{DMA} 、 \mathrm{DPA}$ 与噻吨酮在乙 腈中反应的瞬态吸收光谱(文中没有给出). 在 AN 与 三重态噻吨酮反应中并没有观察到阴阳离子自由基 生成, 只观察到有噻吨酮加氢自由基生成, 这可能与 Schnabel 等 ${ }^{[5}$ 报道的 AN 与三重态噻吨酮反应中阴 阳离子自由基产率较低有关. 在 DMA、DPA 与三重 态噻吨酮反应的瞬态吸收光谱中观察到阴阳离子自 由基和噻吨酮加氢自由基, 这与 Yates ${ }^{\left[{ }^{[b}\right.} 、$ Scigalski ${ }^{[16]}$ 等人得到的结果相同. 一般说来, 电子转移后紧跟着 质子转移的发生, 据此推断出噻吨酮与以上胺类 (TPA、DABCO 除外)发生了以下反应:

$$
\begin{gathered}
{ }^{3} \mathrm{TX}^{*}+\mathrm{DH} \rightarrow{ }^{3}\left(\mathrm{TX}^{-} \cdots \mathrm{DH}^{+}\right) \\
\downarrow \\
{ }^{3}\left(\mathrm{TXH}^{\bullet} \cdots \mathrm{DX}^{\bullet}\right) \rightarrow \mathrm{TXH}^{\bullet}+\mathrm{DH}^{+} \cdot
\end{gathered}
$$

\section{4 三重态噻吨酮与酚类的电子转移和氢转移反应} 噻吨酮与酚类的反应前人的研究涉及较少. 本 实验中我们得到了噻吨酮与几种酚在脱氧乙腈中的 瞬态吸收光谱. 如图 4(a)所示, 是噻吨酮与对苯二酚 在乙腈中的瞬态吸收光谱, 可以看到光脉冲 $0.4 \mu \mathrm{s}$ 后, 噻吨酮三重态很快生成, 随着时间延长至 $1.0 \mu \mathrm{s}$ 时, 对苯二酚对噻吨酮三重态有明显的猝灭, 伴随着 $620 \mathrm{~nm}$ 处吸收峰的衰减, 在 403、420、450-500 nm 处分别生成了三个新的吸收峰. $403 \mathrm{~nm}$ 的峰应归属 为对苯二酚自由基 ${ }^{[2]}, 420 \mathrm{~nm}$ 的峰归属为噻吨酮加 氢自由基, 根据 Yates 等回在苯中得到的信息, 我们
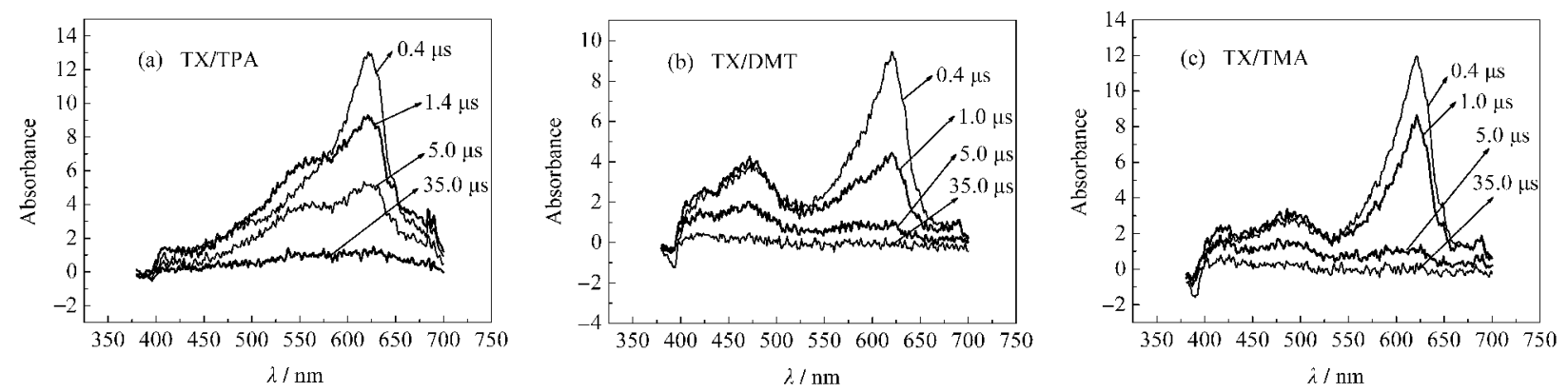

图 $3355 \mathrm{~nm}$ 激光照射脱氧乙腈中 TX 含有(a) TPA, (b) DMT 及(c) TMA 时的瞬态吸收光谱

Fig.3 Transient absorption spectra of TX in the presence of (a) TPA, (b) DMT, and (c) TMA irradiated with $355 \mathrm{~nm}$ laser pulse in deoxygen acetonitrile $c_{\mathrm{TX}}=0.4 \mathrm{mmol} \cdot \mathrm{L}^{-1}, c_{\mathrm{TPA}}=0.19 \mathrm{mmol} \cdot \mathrm{L}^{-1}, c_{\mathrm{DMI}}=0.14 \mathrm{mmol} \cdot \mathrm{L}^{-1}, c_{\mathrm{TMA}}=0.13 \mathrm{mmol} \cdot \mathrm{L}^{-1}$ 

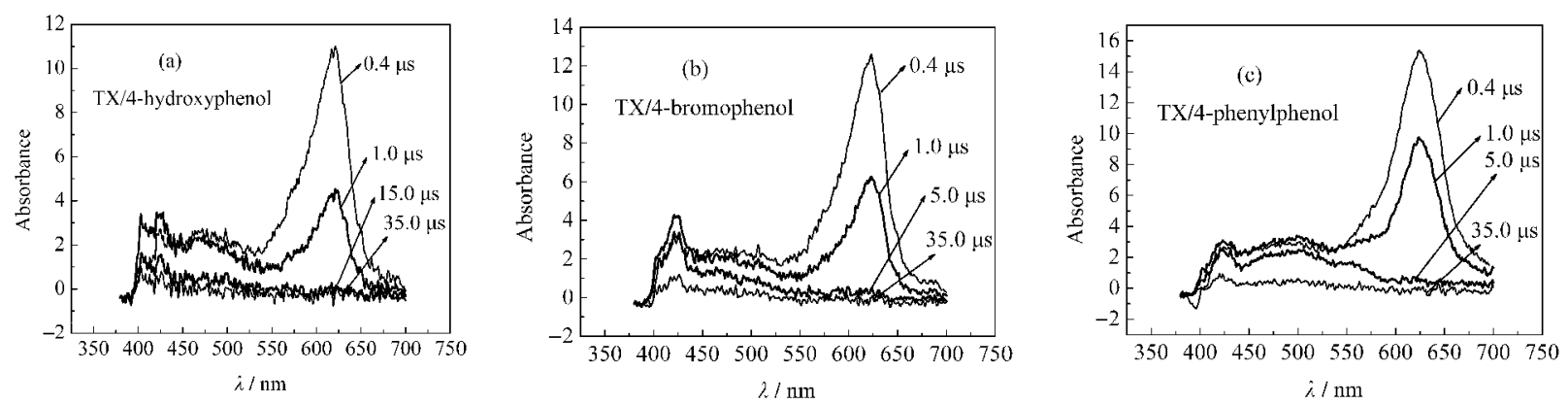

图 $4355 \mathrm{~nm}$ 激光照射脱氧乙腈中 TX 含有(a)对苯二酚, (b) 4-溴苯酚及(c) 4-苯基苯酚时的瞬态吸收光谱

Fig.4 Transient absorption spectra of TX in the presence of (a) 4-hydroxyphenol, (b) 4-bromophenol, and (c) 4-phenylphenol irradiated with $355 \mathrm{~nm}$ laser pulse in deoxygen acetonitrile $c_{\mathrm{TX}}=0.4 \mathrm{mmol} \cdot \mathrm{L}^{-1}$, $=0.25 \mathrm{mmol} \cdot \mathrm{L}^{-1}, c_{\mathrm{b}}$ $=0.41 \mathrm{mmol} \cdot \mathrm{L}^{-1}$, $\mathrm{ol}=0.28 \mathrm{mmol} \cdot \mathrm{L}^{-1}$

把450-500 nm 的吸收归属为噻吨酮加氢自由基, 只 不过此处的吸光系数小于 $420 \mathrm{~nm}$ 处的. 图 4(b) 和图 4(c) 分别为噻吨酮与 4-溴苯酚和 4-苯基苯酚的瞬 态吸收光谱. 与图 4(a)类似, 图 4(b)中随着噻吨酮三 重态吸收峰的衰减, 在 420 和 450-500 nm 处产生 了两个吸收峰, 根据前人报道的 4-溴苯酚自由基特 征吸收峰为 $421 \mathrm{~nm}^{[26]}$, 我们认为此处 $420 \mathrm{~nm}$ 的峰 为 4-溴苯酚自由基与噻吨酮加氢自由基的混合吸 收峰. 图 4(c)中得到了 420 和 $500 \mathrm{~nm}$ 两个吸收峰, $500 \mathrm{~nm}$ 处的吸收峰较宽, 我们把它归属为噻吨酮加 氢自由基在 450-500 nm 处的吸收和 4-苯基苯酚自 由基的吸收, 文献报道 ${ }^{[26]}$, 4-苯基苯酚自由基的特征 吸收是 $502 \mathrm{~nm}$. 所以, DMT 和 TMA 阳离子自由基 出现偏移, 应归因于混人了噻吨酮加氢自由基在 $450-500 \mathrm{~nm}$ 的吸收.

间苯二酚, 4-甲氧基苯酚, 4-叔丁基苯酚, 苯酚, 4-氯苯酚与以上几种酚反应过程类似. 由于只观察

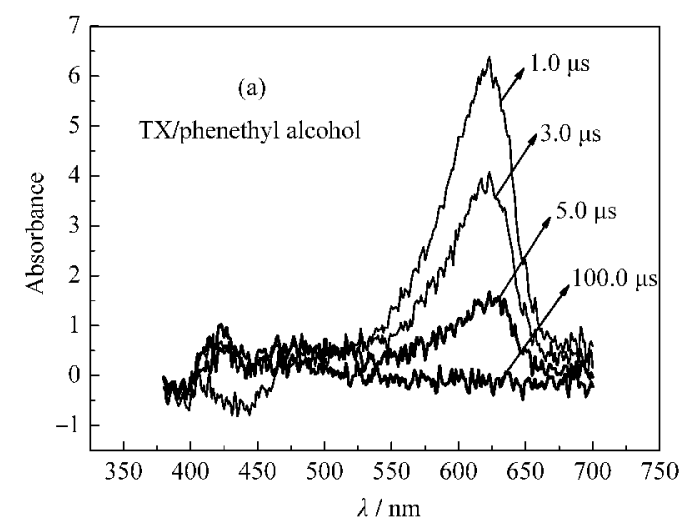

到噻吨酮加氢自由基而没有观察到噻吨酮阴离子自 由基, 所以判断三重态的噻吨酮与酚类并没有发生 电子转移, 而是通过碰撞和库仑作用生成具有电荷 转移性质的激基络合物(exciplex), 随后解离生成噻 吨酮加氢自由基和相应的酚氧自由基 ${ }^{[27]}$. 以上过程 可表示如下:

$$
\begin{aligned}
{ }^{3} \mathrm{TX}^{*}+\mathrm{DOH} \rightarrow 3 & \left(\mathrm{TX}^{\delta-} \cdots \mathrm{DOH}^{\delta+}\right) \rightarrow^{3} \\
\left(\mathrm{TXH}^{\bullet} \cdot \cdots \mathrm{DO}^{\bullet}\right) \rightarrow \mathrm{TXH}^{\bullet}+\mathrm{DO} &
\end{aligned}
$$

\section{5 三重态噻吨酮与醇类发生的氢转移反应}

光诱导醌类、酮类和醇类的反应已有广泛的研 究. 噻吨酮在乙醇、甲醇溶剂中的瞬态吸收光谱也有 报道 ${ }^{[15]}$. 本实验在乙腈中加人苯乙醇得到时间分辨 的瞬态吸收光谱, 如图 5(a)所示, 可以看到随着 620 $\mathrm{nm}$ 处吸收的减弱, 在 420 和 450-500 nm 处有两个 吸收峰生成, 这两个峰归属为噻吨酮加氢自由基, 由 此推断三重态噻吨酮与苯乙醇发生了氢转移反应. 图 5(b)给出了 $420 \mathrm{~nm}$ 处苯甲醇与噻吨酮在乙腈中

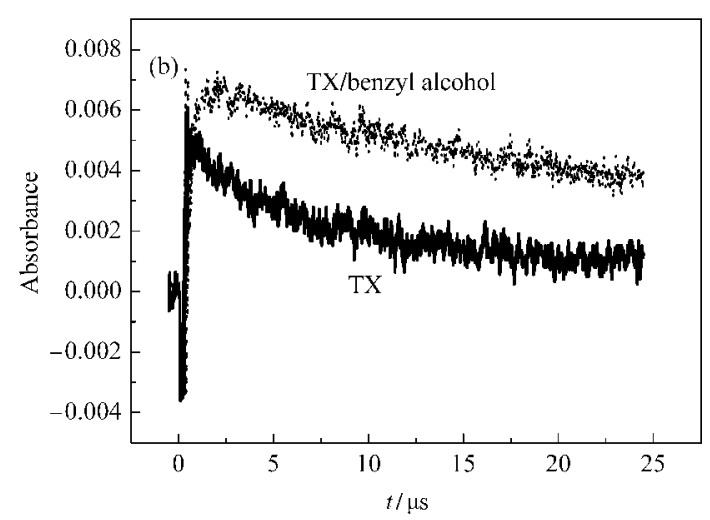

图 $5355 \mathrm{~nm}$ 激光照射脱氧乙腈中(a) TX 含有 2-苯乙醇时的瞬态吸收光谱及(b) TX(实线), TX/苯甲醇(点) $420 \mathrm{~nm}$ 的 衰减曲线

Fig.5 (a) Transient absorption spectra of TX in the presence of phenethyl alcohol and (b) decay time profiles of TX (solid), TX/benzyl alcohol (dot) at $420 \mathrm{~nm}$ irradiated with $355 \mathrm{~nm}$ laser pulse in deoxygen acetonitrile $c_{\mathrm{TX}}=0.4 \mathrm{mmol} \cdot \mathrm{L}^{-1}, c_{\text {phenethyl alcohol }}=2.78 \mathrm{~mol} \cdot \mathrm{L}^{-1}, c_{\text {benzyl alcohol }}=2.42 \mathrm{~mol} \cdot \mathrm{L}^{-1}$ 
的衰减曲线, 和纯的噻吨酮相比, 此处明显有噻吨酮 加氢自由基生成.

同为氢转移反应, 醇类猝灭噻吨酮三重态的速 率常数与酚类相比小了 4-5 个数量级(表 1 和表 2 ), 推断两者经历了不同的氢转移机理. 酚的氧化电势 较小, 容易产生激基复合物, 而醇类不易发生此类反

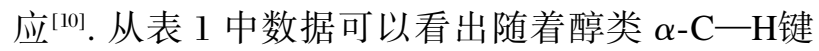
能增大, 猝灭速率常数逐渐减小, 并且 $\alpha-\mathrm{C}-\mathrm{H}$ 键与 醇中其他 $\mathrm{C}-\mathrm{H}$ 键相比有较低的键能, 也最容易被 抽取. 由此推断醇类与噻吨酮发生了如下反应:

$$
\begin{aligned}
& { }^{3} \mathrm{TX}^{*}+\mathrm{DCH}_{2}-\mathrm{OH} \rightarrow{ }^{3}\left(\mathrm{TXH}^{\bullet} \cdots \mathrm{DC} \mathrm{H}-\mathrm{OH}\right) \rightarrow \\
& \mathrm{TXH}^{\bullet}+\mathrm{DC} \dot{\mathrm{C}}-\mathrm{OH}
\end{aligned}
$$

\section{6 热力学结果讨论}

为了进一步证明以上结论的可信性, 我们根据 Rehm-Weller 方程 ${ }^{[28]}$ 求算了上述反应的自由能变 $\Delta G$, 以期得到 $\Delta G$ 与反应猝灭速率常数之间的关系. Rehm-Weller 方程的表达式如下:

$$
\Delta G=E_{\mathrm{ox}}-E_{\mathrm{red}}-q^{2} / \varepsilon r-E_{\mathrm{T}}
$$

其中, $E_{\mathrm{ox}}$ 和 $E_{\mathrm{red}}$ 分别是电子给体的氧化电势和噻吨 酮的还原电势, 酚类 $\left(v s\right.$ 二茂铁标准电极 ${ }^{[18]}$, 二茂铁 标准电极电势 $(v s \mathrm{SCE})$ 为 $\left.0.307 \mathrm{~V}^{[29]}\right)$ 、胺类 $(v s$ 饱和 甘录电极 $(\mathrm{SCE}))^{[30(\mathrm{a}-\mathrm{d})]}$ 的氧化电势列于表 2 和表 3 中. 噻吨酮在乙腈中的还原电势为-1.66 V (vs SCE) ${ }^{[6]}$. $q^{2} / \varepsilon r$ 是产物离子在溶剂中遭遇距离的库仑能量 项, 纯乙腈溶液中此项为 $0.055 \mathrm{eV} . E_{\mathrm{T}}$ 为光敏剂激 发三重态的能量 $(\mathrm{eV})$. 对噻吨酮来说, $E_{\mathrm{T}}$ 为 $274.4 \mathrm{~kJ}$. $\mathrm{mol}^{-1[31]}$. 由此得出的 $\Delta G$ 列于表 2 和表 3 中. 对于胺 类来说, $\Delta G<0$ 可以发生电子转移反应, 实验中也观 察到了相应的阴阳离子自由基产生, 猝灭速率常数 随着 $\Delta G$ 的增大逐渐减小, 电子转移影响了猝灭的 速率. 但是对 TPA 和 DABCO 来说, 猝灭速率常数 却不符合自由能变化的规律, 从胺类与三重态噻吨 酮发生反应的机理来看, 这两种胺只发生电子转移

\section{表 1 醇类猝灭 $\mathrm{TX}$ 三重态的速率常数 $\boldsymbol{k}_{\mathrm{q}}$ 及醇类 $\boldsymbol{\alpha}-\mathrm{C}-\mathrm{H}$ 键能 $\left(\boldsymbol{E}_{\mathbf{B}}\right)$}

Table 1 Quenching rate constants $\left(k_{\mathrm{q}}\right)$ for the triplets

\begin{tabular}{|c|c|c|c|}
\hline \multirow{2}{*}{ Quencher } & \multicolumn{2}{|c|}{$10^{-6} k_{\mathrm{q}} /\left(\mathrm{L} \cdot \mathrm{mol}^{-1} \cdot \mathrm{s}^{-1}\right)$} & \multirow{2}{*}{$\frac{E_{\mathrm{B}}(\alpha-\mathrm{C}-\mathrm{H})^{[19}}{\left(\mathrm{kJ} \cdot \mathrm{mol}^{-1}\right)}$} \\
\hline & TX & $\mathrm{XT}^{[20]}$ & \\
\hline benzyl alcohol & 0.52 & 97 & 366.3 \\
\hline phenethyl alcohol & 0.18 & & 369.5 \\
\hline ethanol & 0.033 & 0.44 & 400.1 \\
\hline methanol & 0.016 & & 411.0 \\
\hline
\end{tabular}
of TX by alcohols and the bonding energy $\left(E_{\mathrm{B}}\right)$ of $\boldsymbol{\alpha}$-C-H in alcohols
表 2 酚类猝灭 $\mathbf{T X}$ 三重态的速率常数 $\boldsymbol{k}_{\mathrm{q}}$, 酚类在乙腈中的 氧化电势 $\boldsymbol{E}_{\mathrm{ox}}$ ( $v s$ 二茂铁标准电极), $\mathrm{TX}$ 三重态与酚类反应的 自由能变 $\Delta G$ 及酚阳离子的酸性 $\left(\mathrm{p} K_{\mathrm{HA}^{+}}\right)$

Table $2 k_{\mathrm{q}}$ for the triplets of TX by phenols, oxidation potentials $\left(E_{0 x} v s\right.$ ferrocenium/ferrocene) of phenols in acetonitrile, free energy changes $(\Delta G)$ for the triplets

\begin{tabular}{|c|c|c|c|c|c|c|}
\hline \multirow{2}{*}{ Quencher } & \multicolumn{3}{|c|}{$10^{-9} k_{\mathrm{q}} /\left(\mathrm{L} \cdot \mathrm{mol}^{-1} \cdot \mathrm{s}^{-1}\right)$} & \multirow{2}{*}{$E_{\text {ox }} / \mathrm{V}^{[18]}$} & \multirow{2}{*}{$\Delta G / \mathrm{eV}$} & \multirow{2}{*}{$\mathrm{p} K_{\mathrm{HA}^{+}}{ }^{118}$} \\
\hline & $\mathrm{XT}^{[20]}$ & TX & $\mathrm{FL}^{[20]}$ & & & \\
\hline 4-hydroxyphenol & & 5.3 & & 1.57 & 0.642 & -5.5 \\
\hline 3-hydroxyphenol & 4.7 & 3.7 & 0.18 & & & \\
\hline 4-methoxyphenol & & 3.2 & & 1.67 & 0.742 & -4.7 \\
\hline 4-phenylphenol & & 2.8 & & 1.86 & 0.932 & -5.7 \\
\hline 4-t-butylphenol & & 2.7 & & 2.00 & 1.07 & -7.2 \\
\hline phenol & 2.1 & 1.8 & 0.033 & 2.10 & 1.17 & -8.1 \\
\hline 4-bromophenol & & 2.1 & & 2.18 & 1.25 & -8.8 \\
\hline 4-chlorophenol & & 2.6 & & 2.23 & 1.30 & -9.9 \\
\hline
\end{tabular}
of TX by phenols, the acidities of the phenol cations $\left(\mathrm{p} K_{\mathrm{HA}^{+}}\right)$

而没有质子转移, 质子转移会加速噻吨酮三重态的 猝灭, 导致 DABCO 和 TPA 猝灭速率显著变小. 对 于酚类来说, $\Delta G>0$ 不能发生电子转移反应, 实验中 我们也没有观察到相应的阴阳离子自由基, 猝灭速 率常数随着 $\Delta G$ 的增大先减小后增大, 究其原因可 能是随着酚阳离子酸性 $\left(\mathrm{p} K_{\mathrm{HA}^{+}}\right)$(表 2)逐渐增大, 带有 部分正电荷的酚供氢能力逐渐增强, 除了电荷作用, 抽氢也对猝灭起较大作用. 二酚的猝灭速率常数都 高于其他苯酚的衍生物, 这与之前报道的分子中质 子氢的数目越多, 氢转移反应活性越大是一致的 ${ }^{[10]}$. 由于对苯二酚、间苯二酚的 Hammett 常数(以苯酚 为参照)分别为 -0.37 和 $0.12^{[23]}$, 则对苯二酚中氧的 电子云密度高于间苯二酚, 有更强的供电子能力, 我

表 3 胺类猝灭 $\mathbf{T X}$ 三重态的速率常数 $\boldsymbol{k}_{\mathrm{q}}$, 胺类在乙腈中 的氧化电势 $E_{\mathrm{ox}}(v s \mathrm{SCE}), \mathrm{TX}$ 三重态与胺类反应的自由能 变 $\Delta \boldsymbol{G}$

Table $3 k_{\mathrm{q}}$ for the triplets of TX by amines, $E_{\text {ox }}(v s$ SCE) of amines in acetonitrile, $\Delta G$ for the triplets of TX by amines

\begin{tabular}{lccccc}
\hline \multirow{2}{*}{ Quencher } & \multicolumn{3}{c}{$10^{-9} k_{\mathrm{q}} /\left(\mathrm{L} \cdot \mathrm{mol}^{-1} \cdot \mathrm{s}^{-1}\right)$} & & \\
\cline { 2 - 6 } & $\mathrm{XT}^{[20]}$ & $\mathrm{TX}$ & $\mathrm{FL}^{[20]}$ & $E_{\text {ox }} / \mathrm{V}(v s \mathrm{SCE})$ & $\Delta G / \mathrm{eV}$ \\
\hline DMT & 16 & 8.1 & 4.8 & $0.65^{[13]}$ & -0.585 \\
TMA & & 7.7 & & $0.74^{[13]}$ & -0.495 \\
DMA & 11 & 6.7 & 2.8 & $0.81^{[30 a]}$ & -0.425 \\
DPA & & 6.5 & & $0.84^{[306]}$ & -0.395 \\
AN & & 5.9 & & $0.98^{[30 c]}$ & -0.255 \\
DABCO & & 5.9 & & $0.57^{[30 \mathrm{~d}]}$ & -0.665 \\
TPA & & 1.8 & & $0.85^{[25]}$ & -0.385 \\
\hline
\end{tabular}

DMT: dimethyl-p-toluidine; TMA: 3,5, $N, N$-tetramethylaniline; DMA: $N, N$-dimethylaniline; DPA: diphenylamine; AN: aniline; DABCO: 1,4-diazobicyclo[2.2.2]octane; TPA: triphenylamine; SCE: saturated calomel electrode 
<smiles>O=c1c2ccccc2oc2ccccc12</smiles>

XT<smiles></smiles>

TX<smiles>O=C1c2ccccc2-c2ccccc21</smiles>

FL

图 $6 \mathrm{XT} 、 \mathrm{TX} 、 \mathrm{FL}$ 的分子结构

Fig.6 Molecular configurations of XT, TX and FL

们得到对苯二酚猝灭噻吨酮三重态的速率常数大于 间苯二酚, 这也是符合电子转移的反应机理的.

\section{7 与胺类、酚类、醇类和呫吨酮、芴酮三重态 反应 ${ }^{[20]}$ 的比较}

呫吨酮、噻吨酮、芴酮是分子结构比较类似的三 个分子, 如图 6 所示, 连接两个苯环的分别是氧原 子、硫原子和单键. 氧原子和硫原子电负性分别为 3.44 和 2.58 , 均表现出一定的吸电子能力 ${ }^{[32]}$, 使激发 双键氧原子的得电子能力加强, 从表 $2 、 3$ 中看到速 率常数按照呫吨酮、噻吨酮、芴酮的顺序逐渐减小, 进一步证明了电子或者电荷转移影响反应速率常数 的机理. 噻吨酮与醇类反应的速率常数比呫吨酮小 2 个数量级(表 1 ), 可能由于硫原子吸电子能力弱于氧 原子, 使双键氧原子对氢原子的作用力小于呫吨酮, 减慢 $\mathrm{C}-\mathrm{H}$ 键的断裂.

\section{3 结 论}

本实验得到了 TPA、DMT、TMA、4-苯基苯酚、 对苯二酚、4-溴苯酚、2-苯乙醇与三重态噻吨酮反应 的瞬态吸收光谱, 通过光谱上自由基吸收峰的时间 演变及速率常数的变化, 推断出如下反应机理: 三 重态噻吨酮与无活泼氢的胺类发生电子转移反应, 与有活泼氢的胺类发生电子-质子转移反应. 猝灭速 率常数随着 $\Delta G$ 的增大逐渐变小, TPA、DABCO 较 小的猝灭速率常数可归因于相关的反应缺少质子转 移的影响. 与酚类反应的猝灭速率常数随着 $\Delta G$ 的 增大先减小后增大, 可以解释为发生电子转移后又 发生了氢转移反应, 由于酚阳离子酸性逐渐增大, 其 供氢能力逐渐增强, 导致猝灭速率常数逐渐变大. 与 醇类反应的猝灭速率常数随着醇类 $\alpha-\mathrm{C}-\mathrm{H}$ 键能的 增大而减小, 表明发生了直接的氢转移反应. 比较胺 类、酚类、醇类分别猝灭 XT、TX、FL 三重态反应的 结果可知, 这些反应有相似的电子或电荷转移机理, 但由于分子结构差异性的影响, 分子中含有吸电子 能力较强的原子猝灭速率常数较大, 即相关反应的 猝灭速率常数按照 XT、TX、FL 的顺序逐渐减小.

\section{References}

1 Morlet-Savary, F.; Ley, C.; Jacques, P.; Wieder, F.; Fouassier, J. P. J. Photochem. Photobiol. A-Chem., 1999, 126: 7

2 Allonas, X.; Ley, C.; Bibaut, C.; Jacques, P.; Fouassier, J. P. Chem. Phys. Lett., 2000, 322: 483

3 Okano, L. T.; Barros, T. C.; Chou, D. T. H.; Bennet, A. J. J. Phys. Chem. B, 2001, 105: 2122

4 Satzger, H.; Schmidt, B.; Root, C.; Zinth, W.; Fierz, B.; Krieger, F.; Kiefhaber, T.; Gilch, P. J. Phys. Chem. A, 2004, 108: 10072

5 Zhu, Q. Q.; Schnabel, W. J. Chem. Soc. Faraday Trans., 1991, 87: 1531

6 Yates, S. F.; Schuster, G. B. J. Org. Chem., 1984, 49: 3349

7 Inbar, S.; Linschitz, H.; Cohen, S. G. J. Am. Chem. Soc., 1980, 102: 1419

8 Guttenplan, J. B.; Cohen, S. G. J. Am. Chem. Soc., 1972, 94: 4040

9 Simon, J. D.; Peters, K. S. J. Am. Chem. Soc., 1981, 103: 6403

10 Wang, J. T.; Pan, Y.; Zhang, L. M.; Yu, S. Q. Chinese Journal of Chemical Physics, 2007, 20: 395 [王金婷, 潘 洋, 张立敏, 俞书勤. 化学物理学报, 2007, 20: 395]

11 Stevenson, J. P.; DeMaria, D.; Reilly, D.; Purvis, J. D.; Graham, M. A.; Lockwood, G.; Drozd, M.; O'Dwyer, P. J. Cancer Chemother. Pharmacol., 1999, 44: 228

12 Izbicka, E.; Lawrence, R.; Davidson, K.; Rake, J. B.; VonHoff, D. D. Invest. New Drugs, 1999, 16: 221

13 Pan, Y.; Fu ,Y.; Liu, S. X.; Yu, H. Z.; Gao, Y. H.; Guo, Q. X.; Yu S. Q. J. Phys. Chem. A, 2006, 110: 7316

14 Dalton, J. C.; Montgomery, F. C. J. Am. Chem. Soc., 1974, 96: 6230

15 Ferreira, G. C.; Schmitt, C. C.; Neumann, M. G. J. Braz. Chem. Soc., 2006, 17: 905

16 Scigalski, F.; Paczkowski, J. Macromol. Chem. Phys., 2008, 209 1872

17 Das, D.; Nath, D. N. J. Phys. Chem. A, 2008, 112: 11619

18 Bordwell, F. G.; Cheng, J. P. J. Am. Chem. Soc., 1991, 113: 1736

19 Denisov, E. T.; Denisova, T. G. Handbook of antioxidants. Boca Raton: CRC Press, 2000: 24

20 Wang, J. T. Laser flash photolysis studies on some organic molecules [D]. Hefei: University of Science and Technology of China, 2009 [王金婷. 若干有机分子的激光闪光光解研究[D]. 合肥: 中国科学技术大学, 2009]

21 Pan, Y.; Sheng, Z. Y.; Ye, X. D.; Ao, Z.; Yu, S. Q. J. Photochem. Photobiol. A-Chem., 2005, 174: 98

22 Pan, Y.; Sheng, Z. Y.; Li, J.; Dai, J. H.; Chu, G. S.; Yu, S. Q. Acta 
Chim. Sin., 2004, 62: 1293 [潘 洋, 盛震宇, 李 江, 戴静华, 储高升, 俞书勤. 化学学报, 2004, 62: 1293]

23 Murov, S. L.; Carmichael, I.; Hug, G. Handbook of photochemistry. 2nd ed. New York: Marcel Dekker, 1993: 111, 346-348

24 Corrales, T.; Peinado, C.; Catalina, F.; Neumann, M. G.; Allen, N. S.; Rufs, A. M.; Encinas, M. V. Polymer, 2000, 41: 9103

25 Bartholomew, R. F.; Davidson, R. S.; Lambeth, P. F.; Mckellar, J. F.; Turner, P. H. J. Chem. Soc. Perkin Trans., 1972, 2: 577

26 Das, P. K.; Encinas, M. V.; Steenken, S.; Scaiano, J. C. J. Am. Chem. Soc., 1981, 103: 4162

27 Yoshihara, T.; Yamaji, M.; Itoh, T.; Shizuka, H.; Shimokage, T.; Tero-Kubota, S. Phys. Chem. Chem. Phys., 2000, 2: 993

28 Rehm, D.; Weller, A. Isr. J. Chem., 1970, 8: 259
29 Bard, A. J.; Faulkner, L. R. Electrochemical methods, fundamentals and applications. New York: John Wiley, 1980: 701

30 (a) Nocera, D. G.; Gray, H. B. J. Am. Chem. Soc., 1981, 103: 7349 (b) Dvorak, V.; Nemec. I.; Zyka, J. J. Microchem. J., 1967, 12: 99 (c) Pan, Y.; Tang, W. J.; Yu, T. Q.; Wang, J. T.; Fu, Y.; Wang, G. W.; Yu, S. Q. J. Lumin., 2007, 126: 421

(d) Nelsen, S. F.; Hinz, P. J. J. Am. Chem. Soc., 1972, 94: 7114

31 Herkatroeter, W. G.; Lamula, A. A.; Hammond, G. S. J. Am. Chem. Soc., 1964, 86: 4537

32 Cao, X. Z.; Song, T. Y.; Wang, X. Q. Inorganic chemistry. 3rd ed. Beijing: Higher Education Press, 1994: 114-116［曹锡章, 宋天 佑, 王杏乔. 无机化学. 北京: 高等教育出版社, 1994: 114-116] 